TRABAJOS ORIGINALES

Rev Obstet Ginecol Venez. 2021; 81 (1): 5-12

https://doi.org/10.51288/00810104

\title{
Utilidad diagnóstica de la relación plaquetas/linfocitos en embarazadas con preeclampsia
}

\author{
Drs. Eduardo Reyna-Villasmil, ${ }^{1}$ Jorly Mejia-Montilla, ${ }^{2}$ Nadia Reyna-Villasmil, ${ }^{2}$ Duly Torres-Cepeda, ${ }^{1}$ \\ Andreina Fernández-Ramírez. ${ }^{2}$
}

\begin{abstract}
RESUMEN
Objetivo: Establecer la utilidad diagnóstica de la relación plaquetas/linfocitos en embarazadas con preeclampsia. Métodos: Se realizó un estudio de casos y controles en el Hospital Central "Dr. Urquinaona”, Maracaibo, Venezuela. Se seleccionó un total de 180 embarazadas. Se incluyeron 90 preeclámpticas como grupo de estudio (grupo A) y un grupo de control seleccionado por tener edad e indice de masa corporal similares al grupo de estudio, que consistió en 90 embarazadas normotensas sanas (grupo B). Se determinaron las características generales, valores de la relación plaquetas/linfocitos y eficacia diagnóstica.

Resultados: Las pacientes del grupo A presentaron valores más bajos de plaquetas y linfocitos comparado con las pacientes del grupo $B(p<0,001)$. No obstante, no se encontraron diferencias estadísticamente significativas en la relación plaquetas/linfocitos entre las pacientes del grupo A (115,2 $\pm 32,7)$ y las pacientes del grupo B (122,3 $\pm 23,8$; $p=0,0971)$. Un valor de corte de la relación plaquetas/linfocitos de 117 presentó un valor por debajo de la curva de 0,57, sensibilidad del 47,8\%, especificidad del 50,0\%, valor predictivo positivo del 48,9\% y valor predictivo negativo del 52,2\%, con una exactitud diagnóstica del 48,9\%.

Conclusión: La relación plaquetas/linfocitos no es una herramienta útil en el diagnóstico de preeclampsia, ya que las pacientes con el síndrome presentan valores similares a las embarazadas normotensas.
\end{abstract}

Palabras clave: Relación plaquetas/linfocitos, Preeclampsia, Diagnóstico, Embarazo.

\section{SUMMARY}

Objective: To establish the diagnostic utility of the platelet-lymphocyte ratio in pregnant women with preeclampsia. Methods: A case-control study was carried out at the Hospital Central "Dr. Urquinaona" Maracaibo, Venezuela. A total of 180 pregnant women were selected. Ninety preeclamptic women were included as the study group (group A) and a control group selected for their age and body mass index similar to the study group, which consisted of 90 healthy normotensive pregnant women (group B). The general characteristics, platelet-lymphocyte ratio values and diagnostic efficacy were determined.

Results: Group A patients presented lower platelet and lymphocyte values compared to group B patients $(p<0.001)$. However, no statistically significant differences were found in platelet-lymphocyte ratio between group A patients (115.2 \pm 32.7$)$ and group B patients (122.3 $\pm 23.8 ; p=0$, 0971). A cut-off value of the platelets-lymphocyte ratio of 117 presented a value below the curve of 0.57 , the sensitivity of $47.8 \%$, the specificity of $50.0 \%$, the positive predictive value of $48.9 \%$, and the negative predictive value of $52.2 \%$, with a diagnostic accuracy of $48.9 \%$.

Conclusion: Platelet-lymphocyte ratio is not a useful tool in the diagnosis of preeclampsia, since patients with the syndrome present similar values to normotensive pregnant women.

Keywords: Platelet-lymphocyte ratio, Preeclampsia, Diagnosis, Pregnancy.

\section{INTRODUCCIÓN}

'Servicio de Obstetricia y Ginecología - Maternidad "Dr. Nerio Belloso" Hospital Central "Dr. Urquinaona". Maracaibo, Estado Zulia. ${ }^{2}$ Facultad de Medicina. La Universidad del Zulia. Maracaibo.
La preeclampsia es un trastorno específico del embarazo que está caracterizado por hipertensión y proteinuria, luego de las 20 semanas de gestación, 
en una mujer previamente normotensa. Es una complicación común del embarazo, ya que afecta $3 \%-5 \%$ de todos los embarazos y es responsable de cerca del $12 \%$ de las muertes maternas (1). Aunque la causa es desconocida, existe evidencia de disfunción endotelial, placentación anormal e inflamación sistémica (2). Estos cambios conducen a alteración de la respuesta inmune y a un estado proinflamatorio (3-5). La disfunción endotelial causa vasoconstricción, agregación, adhesión plaquetaria y acentúa la hipercoagulabilidad que resulta en daño hipóxico endotelial (6). Además, existe aumento del recambio plaquetario, acompañado de cambios en el número, volumen y función (7).

La trombosis, inflamación y aterogénesis son eventos fisiopatológicos íntimamente relacionados y tanto las plaquetas como los leucocitos son sus principales efectores celulares. El contaje elevado de plaquetas puede reflejar aumento de la activación plaquetaria, proliferación megacariocítica y trombocitosis relativa (8). Además, los recuentos bajos de linfocitos parecen indicar que la alteración de la respuesta inmunitaria está asociada con resultante adversa en pacientes con trastornos cardiovasculares (9).

La relación plaquetas/linfocitos (RPL), un indicador de la activación plaquetaria, de la función de los linfocitos y de la respuesta inmune, ha generado interés como fuerte predictor de inflamación sistémica en diversas condiciones clínicas. Un valor elevado es un potencial marcador de pronóstico en sujetos con enfermedades cardiovasculares, infarto del miocardio y enfermedad cardiaca coronaria (10). Sin embargo, su valor diagnóstico en los casos de preeclampsia sigue sin estar claro. Por lo tanto, el objetivo del estudio fue establecer la utilidad de la relación plaquetas/linfocitos en el diagnóstico de preeclampsia.

\section{MÉTODOS}

Se realizó un estudio de casos y controles entre enero de 2017 y febrero de 2020 que incluyó mujeres con embarazos simples que fueron atendidas en el Hospital Central "Dr. Urquinaona", Maracaibo, Venezuela. La investigación fue aprobada por el Comité de Ética e Investigación del hospital y se obtuvo consentimiento por escrito de todas las pacientes.

Se incluyó un total de 180 embarazadas de las cuales 90 preeclámpticas representaron los casos (grupo A), seleccionados en forma aleatoria y consecutiva, y 90 embarazadas normotensas sanas (grupo B) que constituyeron el grupo control, seleccionado por tener edad materna e índice de masa corporal, al momento de la selección, similar al grupo de estudio.

Se excluyó a las embarazadas con polihidramnios, hemorragia del tercer trimestre (desprendimiento prematuro de placenta, placenta previa), sospecha de restricción del crecimiento intrauterino del feto (circunferencia cefálica, circunferencia abdominal y longitud del fémur menor del percentil 10 de referencia con confirmación posnatal de peso menor al percentil 10 de referencia), síndrome HELLP, alteraciones de la frecuencia cardiaca fetal, gestaciones múltiples, presencia de infección intrauterina o materna activa, enfermedad hipertensiva crónica (hipertensión antes de las 20 semanas de embarazo). Además fueron excluidas aquellas participantes con enfermedad cardiaca, hematológicas, hepática, renal o sistémica crónica, diabetes mellitus pre- o gestacional, hábito tabáquico, aquellas embarazadas en las cuales no se pudo obtener muestras de sangre y en las que hayan utilizado medicamentos que alteren la concentración de plaquetas o linfocitos (por ejemplo, antihipertensivos, expansores plasmáticos). También se excluyó a las pacientes que se negaron a participar en la investigación.

La preeclampsia se definió como presión arterial sistólica de $140 \mathrm{~mm}$ de $\mathrm{Hg}$ o más, o presión arterial diastólica de $90 \mathrm{~mm}$ de $\mathrm{Hg}$ o más, confirmada en 6 horas o más de diferencia, mientras que la proteinuria 
se definió como 300 mg o más de proteína en una muestra de 24 horas o 1-2 cruces de proteinuria en un examen cualitativo después de las 20 semanas de gestación. La presión sanguínea se midió en posición sentada después de 15 minutos de descanso usando un esfigmomanómetro de mercurio estándar con un manguito de 14 centímetros. Los valores de presión arterial sistólica y diastólica (tomada en relación con el quinto ruido de Korotkoff) fueron ubicados con respecto al punto de $2 \mathrm{~mm}$ de $\mathrm{Hg}$ más cercano. El método palpatorio se utilizó para verificar las lecturas auscultatorias de la presión arterial sistólica. Las presiones arteriales sistólica y diastólica se calcularon del promedio de la presión arterial de cada brazo.

Se recolectaron $10 \mathrm{ml}$ de sangre de la vena antecubital, en todas las pacientes de ambos grupos, para realizar una única determinación al momento del ingreso y antes de la atención del parto e inicio de cualquier tratamiento médico. Estas muestras se colocaron en un tubo de vidrio seco, estéril, almacenado a temperatura ambiente y protegidos de la luz ultravioleta. Posteriormente fueron centrifugados a $1600 \mathrm{rpm}$ por 10 minutos, separados en alícuotas y almacenados a $-70^{\circ} \mathrm{C}$ hasta el momento del análisis.

Las mediciones de hemoglobina se realizaron utilizando un analizador cuantitativo automático de hematología LH75 (Beckman Coulter Inc., ${ }^{\circledR}$ EE.UU.). Las concentraciones séricas de ácido úrico y creatinina también se determinaron por espectrofotometría con un kit comercial (DiaSys Diagnostic Systems, ${ }^{\circledR}$ Alemania) con una sensibilidad de $0,01 \mathrm{mg} / \mathrm{dL}$ y $0,1 \mathrm{mg} / \mathrm{dL}$, respectivamente. La proteinuria se determinó en una muestra de orina de $24 \mathrm{~h}$ y se almacenó a $-20^{\circ} \mathrm{C}$. Los valores se determinaron por medio de una prueba turbidimétrica (Raichem, ${ }^{\circledR}$ EE.UU.). Los coeficientes de variación inter- e intraensayo fueron menores del $5 \%$ y el $7 \%$, respectivamente. Las concentraciones de aspartato aminotransferasa y alanino aminotransferasa se midieron por el método de espectrofotometría usando kits comerciales (DiaSys Diagnostic Systems, ${ }^{\circledR}$
Alemania) con sensibilidad de 4 UI/L y 2 UI/L, respectivamente.

Los recuentos totales y diferenciales de leucocitos y plaquetas se midieron utilizando un analizador de hematología automático Abbott Cell-Dyn 3700 (Abbott Laboratory, ${ }^{\circledR}$ EE.UU). Se usaron recuentos absolutos (células x 103/mL) de células en los análisis. La RPL fue determinada utilizando estos valores.

Los datos se presentan como valores promedios \pm desviación estándar. Se verificó la normalidad de los datos antes del análisis estadístico utilizando la prueba de Kolmogorov-Smirnov. Las comparaciones de las variables continuas entre los dos grupos distribuidas normalmente se compararon usando la prueba t para muestras no relacionadas, mientras que la prueba $U$ de Mann-Whitney se utilizó para aquellas variables con distribución diferente a la normal. Se utilizó la prueba de Pearson o Spearman, dependiendo de la distribución de los datos, para establecer la correlación entre la presión arterial, valores de los parámetros de laboratorio y peso del recién nacido con los valores la RPL. El valor de corte óptimo de la RPL para discriminar entre los grupos de casos y controles se realizó por el análisis del área bajo la curva de la función operador-respuesta como la suma máxima de sensibilidad y especificidad para una prueba significativa. La sensibilidad, especificidad, valores predictivos positivo y negativo se calcularon para el mejor valor de corte. Se consideró $\mathrm{p}<0,05$ como estadísticamente significativa.

\section{RESULTADOS}

Las características de las preeclámpticas (grupo A) y las embarazadas normotensas (grupo B) se muestran en la tabla 1. En el grupo A se diagnosticaron 59 pacientes $(64,8 \%)$ sin criterios de gravedad y 31 pacientes $(34,4 \%)$ con criterios de gravedad o preeclampsia grave. No se encontraron diferencias estadísticamente significativas con relación a la edad materna, edad 
Tabla 1. Características generales

\begin{tabular}{lccc}
\hline $\begin{array}{l}\text { Promedio } \pm \\
\text { desviación estándar }\end{array}$ & $\begin{array}{c}\text { Grupo A } \\
\text { Casos } \\
(\mathrm{n}=90)\end{array}$ & $\begin{array}{c}\text { Grupo B } \\
\text { Controles } \\
(\mathrm{n}=90)\end{array}$ & $p$ \\
\hline $\begin{array}{l}\text { Edad, años } \\
21,9 \pm 2,4\end{array}$ & $22,4 \pm 2,3$ & $\mathrm{~ns}$ \\
$\begin{array}{l}\text { Edad gestacional al } \\
\text { momento del parto, } \\
\text { semanas }\end{array}$ & $38,8 \pm 1,0$ & $38,9 \pm 1,1$ & $\mathrm{~ns}$ \\
$\begin{array}{l}\text { Índice de masa } \\
\text { corporal, Kg/m }\end{array}$ & $30,0 \pm 1,2$ & $29,7 \pm 1,2$ & ns \\
$\begin{array}{l}\text { Presión arterial } \\
\text { sistólica, mm de Hg }\end{array}$ & $150,2 \pm 12,6$ & $104,5 \pm 6,1$ & $<0,001$ \\
$\begin{array}{l}\text { Presión arterial } \\
\text { diastólica, mm de Hg }\end{array}$ & $106,2 \pm 8,3$ & $74,3 \pm 8,0$ & $<0,001$ \\
$\begin{array}{l}\text { Peso del recién } \\
\text { nacido, gramos }\end{array}$ & $2929 \pm 344$ & $3645 \pm 390$ & $<0,001$ \\
\hline
\end{tabular}

gestacional al momento del parto e índice de masa corporal materno $(\mathrm{p}=\mathrm{ns})$, pero sí hubo diferencias estadísticamente significativas en los valores de la presión arterial sistólica, presión arterial diastólica y peso del recién nacido $(\mathrm{p}<0,001)$.
En la tabla 2 se muestran los valores de laboratorio de cada uno de los grupos. Las concentraciones de hemoglobina, transaminasas, creatinina, ácido úrico y proteinuria fueron significativamente más altas en las pacientes del grupo A comparado con las pacientes del grupo B $(\mathrm{p}<0,001)$. Las pacientes del grupo A presentaron valores más bajos de plaquetas y linfocitos $\left(174,6 \times 10^{3} / \mathrm{mL}\right.$ y $2,0 \pm 0,5 \times 10^{3} / \mathrm{mL}$, respectivamente) comparado con las pacientes del grupo B $\left(252,1 \times 10^{3}\right.$ $\mathrm{mL}$ y $\left.2,4 \pm 0,5 \times 10^{3} / \mathrm{mL} ; \mathrm{p}<0,001\right)$. No obstante, no se encontraron diferencias estadísticamente significativas en la RPL entre las pacientes del grupo A $(115,2 \pm 32,7)$ y las pacientes del grupo B $(122,3 \pm$ 23,$8 ; \mathrm{p}=0,0971$; figura 1).

Al correlacionar los valores de la RPL con los valores de presión arterial, se observaron correlaciones débiles, negativas y significativas con los valores de presión arterial sistólica $(\mathrm{r}=-0,155)$ y diastólica $(r=-0,129)$ en forma global $(p<0,05)$. Sin embargo, no se encontró correlación con la presión arterial sistólica y diastólica en cada uno de los grupos de forma individual

Tabla 2. Características de laboratorio

\begin{tabular}{|c|c|c|c|}
\hline Promedio \pm desviación estándar & $\begin{array}{c}\text { Grupo A } \\
\text { Casos } \\
(\mathrm{n}=90)\end{array}$ & $\begin{array}{c}\text { Grupo B } \\
\text { Controles } \\
(\mathrm{n}=90)\end{array}$ & $p$ \\
\hline Hemoglobina, $\mathrm{g} / \mathrm{dL}$ & $11,1 \pm 1,5$ & $10,3 \pm 1,3$ & $<0,001$ \\
\hline Plaquetas, $\mathrm{x} 10^{3} / \mathrm{mm}^{3}$ & $174,6 \pm 40,1$ & $252,1 \pm 30,3$ & $<0,001$ \\
\hline Aspartatoaminotransferasa, UI/L & $90,0 \pm 29,0$ & $22,7 \pm 7,7$ & $<0,001$ \\
\hline Alaninoaminotransferasa, UI/1 & $107,6 \pm 34,6$ & $27,9 \pm 5,4$ & $<0,001$ \\
\hline Creatinina, $\mathrm{mg} / \mathrm{dL}$ & $0,9 \pm 0,1$ & $0,7 \pm 0,1$ & $<0,001$ \\
\hline Ácido úrico, mg/dL & $5,0 \pm 0,6$ & $3,4 \pm 0,3$ & $<0,001$ \\
\hline Proteinuria en 24 horas, g & $3,95 \pm 0,59$ & $0,15 \pm 0,02$ & $<0,001$ \\
\hline Deshidrogenasa láctica, UI/L & $523,9 \pm 131,7$ & $241,0 \pm 76,3$ & $<0,001$ \\
\hline Leucocitos, x $10^{3} / \mathrm{mL}$ & $9,8 \pm 1,7$ & $9,3 \pm 1,4$ & 0,0326 \\
\hline Neutrófilos, x $10^{3} / \mathrm{mL}$ & $7,7 \pm 1,5$ & $6,5 \pm 1,2$ & $<0,001$ \\
\hline Linfocitos, $\times 10^{3} / \mathrm{mL}$ & $2,0 \pm 0,5$ & $2,4 \pm 0,5$ & $<0,001$ \\
\hline Relación plaquetas/linfocitos & $115,2 \pm 32,7$ & $122,3 \pm 23,8$ & 0,0971 \\
\hline
\end{tabular}




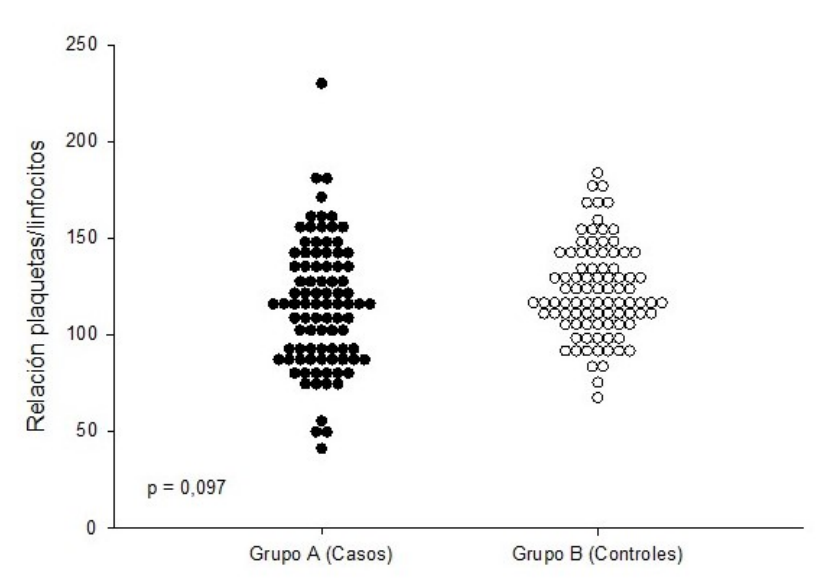

Figura 1. Valores de la relación plaquetas/linfocitos en cada uno de los grupos de estudio.

$(\mathrm{p}=\mathrm{ns})$. Tampoco mostraron correlaciones significativas con ninguno de los otros parámetros de laboratorio en las preeclámpticas $(\mathrm{p}=\mathrm{ns})$. Se encontró correlación moderada, positiva y significativa con el peso del recién nacido en el grupo de casos $(r=-0,348, p<$

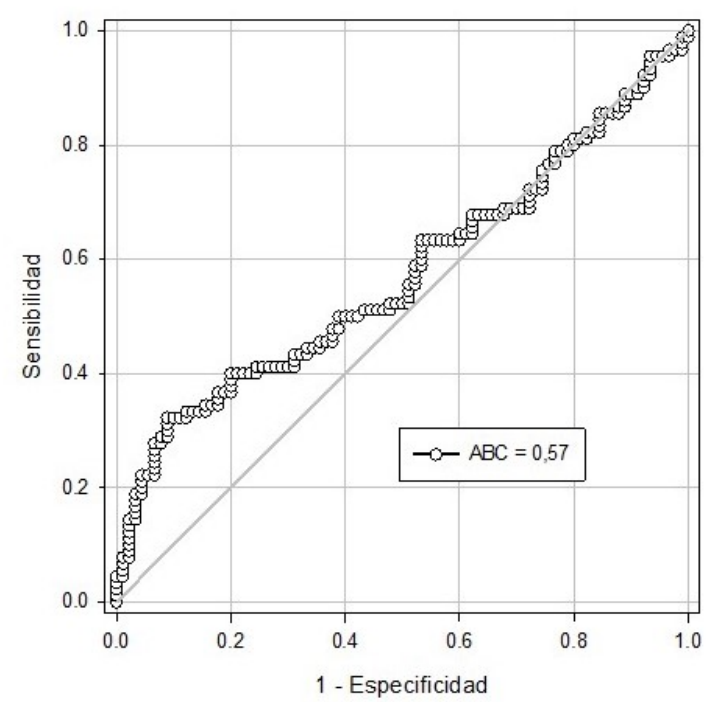

Figura 2. Curva operador-receptor de la relación plaquetas/linfocitos para el diagnóstico de preeclampsia, para un valor de corte de 117.
0,001), pero no se encontró correlación entre estos dos parámetros en el grupo control $(\mathrm{r}=0,104$; $\mathrm{p}=0,330)$.

Al analizar la capacidad del valor de corte de la RPL de 117 en el diagnóstico de preeclampsia (figura 2) se observó que el área bajo la curva fue de 0,57 (intervalo de confianza [IC] del $95 \% ; 0,47-0,66$ ) con sensibilidad del 47,8 \% (IC del $95 \%$; 37,8 - 58,2), especificidad del 50,0\% (IC del 95\%; 39,9-60,1), valor predictivo positivo del 48,9\% (IC del $95 \% ; 38,9$ $-59,1 \%$ ) y valor predictivo negativo del $52,2 \%$ (IC del $95 \% ; 42,0-62,2 \%$ ). La relación de probabilidad positiva fue de 0,962 (IC del $95 \% ; 0,717-1,298$ ) y la relación de probabilidad negativa fue de 1,048 (IC del $95 \% ; 0,783-1,393)$. La exactitud diagnóstica de la prueba fue de $48,9 \%$.

\section{DISCUSIÓN}

Actualmente, uno de los objetivos más importantes en obstetricia es poder identificar en formar efectiva a las preeclámpticas. La determinación de indicadores sensibles y específicos no solo podría identificar a las pacientes con riesgo de desarrollar hipertensión durante el embarazo, sino también la importancia de identificar con precisión o predecir el momento de aparición y proporcionar las intervenciones adecuadas en las preeclámpticas. Los resultados de esta investigación demuestran que los valores de RPL no son útiles para el diagnóstico de preeclampsia, ya que carecen de capacidad de discriminación de la patología.

La preeclampsia es un trastorno importante relacionado con aumento de la morbimortalidad materna y perinatal. Los cambios endoteliales conducen al desarrollo de trombosis microvascular, aumento de la permeabilidad capilar y del tono vascular resultando en la aparición de hipertensión (11). La patogenia tiene dos etapas. En la primera etapa ocurre invasión deficiente de las arterias espirales con disminución del flujo arterial úteroplacentario resultando en perfusión 
inadecuada. Esto conduce a hipoxia y liberación de especies reactivas de oxígeno, que acentúan el estrés oxidativo y la disfunción placentaria (12). Todo esto induce inflamación con liberación de quimiocinas, citocinas proinflamatorias, factores antiangiogénicos y activación de monocitos/neutrófilos (13). La segunda etapa comienza cuando los neutrófilos activados infiltran el tejido vascular materno, produciendo inflamación sistémica materna con activación plaquetaria, vasoconstricción, hipertensión, disfunción endotelial e isquemia de órganos terminales (1214). Por otra parte, existe un cambio hacia un estado proinflamatorio, con mayor alteración de la respuesta inmune $(1,13)$.

Existe evidencia de valores elevados de la RPL asociados con estados inflamatorios y eventos cardiovasculares. La RPL también ha sido considerada como un indicador útil del estado inflamatorio en pacientes con neoplasias (15). Un mayor valor de la relación está asociado con complicaciones en pacientes con enfermedades cardiacas y también es útil como marcador de mortalidad a largo plazo (16).

Varios estudios han investigado los cambios $\mathrm{y}$ aplicabilidad de diferentes índices plaquetarios, en preeclámpticas con resultados contradictorios. Tres estudios previos evaluaron la utilidad diagnóstica y/o pronóstica de la RPL en la preeclampsia. El primer estudio encontró que la RPL era menor en las preeclámpticas con signos de gravedad comparadas con las embarazadas sanas, pero esta diferencia no fue significativa (17). El segundo estudio demostró que, aunque el valor de RPL era significativamente menor en las preeclámpticas, era un mal predictor de la gravedad del síndrome (18). Finalmente, otra investigación encontró diferencias estadísticamente significativas en los valores de la relación entre preeclámpticas y embarazadas normotensas, siendo los valores significativamente menores en las preeclámpticas graves que en las leves. Además, era mejor predictor de preeclampsia comparado con la amplitud de distribución plaquetaria y volumen plaquetario medio (19). En esta investigación no se encontraron diferencias en la RPL entre las preeclámpticas y las embarazadas sanas, por lo que no es útil para diagnóstico.

Las causas de la trombocitopenia en las preeclámpticas son desconocidas, pero aproximadamente $30 \%$ - $50 \%$ de los pacientes con preeclampsia grave presentan disminución del contaje de plaquetas, el cual representa un papel importante en la progresión del síndrome $(20,21)$. Diferentes estudios mostraron que las concentraciones de plaquetas disminuyen en forma significativa en mujeres que desarrollaron preeclampsia, comparado con embazadas sanas (15, 22-24). De igual forma, la preeclampsia también está asociada con aumento del recuento de leucocitos, superior al observado en el embarazo normal, y este aumento es secundario al aumento en el número de neutrófilos acompañado de disminución de la cantidad de linfocitos (25).

Además, la disfunción endotelial en la preeclampsia produce activación intravascular incontrolada de plaquetas con aumento del consumo en la circulación periférica materna $(15,22)$. La depuración plaquetaria aumenta por activación de las plaquetas circulantes a través de activación de la cascada de la coagulación, la adhesión de las plaquetas al endotelio dañado o activado y eliminación de plaquetas recubiertas de anticuerpos a través del sistema reticuloendotelial (26).

Diferentes subtipos de leucocitos están asociados con enfermedades cardiovasculares. Los neutrófilos desempeñan un papel importante en la patogenia de la preeclampsia. Su activación es consecuencia de la exposición a lípidos oxidados secretados por la placenta (14). Además, tienen un papel importante en los procesos inflamatorios, ya que son las primeras células 
sanguíneas de respuesta (10). Existen informes de aumento de la cantidad de linfocitos en preeclámpticas, lo que indicaría un estado proinflamatorio (27). La hipoxia tisular en las preeclámpticas provoca secreción de eritropoyetina y estimulación de la médula ósea, lo que resulta en aumento del número de megacariocitos y plaquetas. Las plaquetas y los neutrófilos activados atraviesan el espacio intervelloso y provocan disfunción endotelial (28). Además, la interacción células endoteliales/plaquetas provoca liberación de sustancias inflamatorias e induce la migración y adhesión de los leucocitos $(11,29)$.

\section{CONCLUSIÓN}

Los resultados de la investigación sugieren que la relación plaquetas/linfocitos no es una herramienta útil en el diagnóstico de preeclampsia, ya que las pacientes con el síndrome no presentan valores diferentes a las embarazadas normotensas.

\section{REFERENCIAS}

1. Lokki AI, Heikkinen-Eloranta JK, Laivuori H. The Immunogenetic Conundrum of Preeclampsia. Front Immunol. 2018; 9:2630. doi: 10.3389/ fimmu.2018.02630. PMID: 30483272; PMCID: PMC6243043.

2. Tangren JS, Thadhani R. Novel Preeclampsia Diagnostics and Real-World Applications. Hypertension. 2019; 74(4):740-742. doi: 10.1161/ HYPERTENSIONAHA.119.12908. Epub 2019 Aug 12. PMID: 31401879.

3. Negishi Y, Takahashi H, Kuwabara Y, Takeshita T. Innate immune cells in reproduction. J Obstet Gynaecol Res. 2018; 44(11):2025-2036. doi: 10.1111/jog. 13759. Epub 2018 Jul 29. PMID: 30058156.

4. Molina Vílchez R, Herrera J. Angiopatía de la gestación: el quijote y la preeclampsia. Rev Obstet Ginecol Venez [Internet]. 2001 [consultado junio de 2020]; 61(2): 73 - 75. Disponible en: http://www.sogvzla. org.ve/sogvzla20186/cms/svcobtenerpdfrevista. php?id $=0000000049 \&$ tipo $=$ normal\&fila $=2$

5. Contreras F, Martínez J, Fouillioux C, Colmenares
Y, Guevarra E, Torres D, et al. Endotelio y trastornos hipertensivos en el embarazo. Rev Fac Med (Caracas) [Internet]. 2002 [consultado junio 2020]; 25(1): 121 - 129. Disponible en: http://ve.scielo.org/scielo.php?script=sci arttext\&pid=S0798-04692002000100031

6. Gilani SI, Weissgerber TL, Garovic VD, Jayachandran M. Preeclampsia and Extracellular Vesicles. Curr Hypertens Rep. 2016; 18(9):68. doi: 10.1007/s11906016-0678-x. PMID: 27590522; PMCID: PMC5010622.

7. Reddy SG, Rajendra PrasadCSB. Significance of platelet indices as severity marker in nonthrombocytopenic preeclampsia cases. J Lab Physicians. 2019; 11(3):186191. doi: 10.4103/JLP.JLP_161_18. PMID: 31579247; PMCID: PMC6771321.

8. Katz OB, Brenner B, Horowitz NA. Thrombosis in vasculitic disorders-clinical manifestations, pathogenesis and management. Thromb Res. 2015; 136(3):504-12. doi: 10.1016/j.thromres.2015.07.016. Epub 2015 Jul 15. PMID: 26220271.

9. Oylumlu M, Oylumlu M, Arslan B, Polat N, Özbek $\mathrm{M}$, Demir M, et al. Platelet-to-lymphocyte ratio is a predictor of long-term mortality in patients with acute coronary syndrome. Postepy Kardiol Interwencyjnej. 2020; 16(2):170-176. doi: 10.5114/aic.2020.95859. Epub 2020 Jun 23. PMID: 32636901; PMCID: PMC7333190.

10. Yan L, Hu ZD. Red Blood Cell Distribution Width, Neutrophil-to-Lymphocyte Ratio, and InHospital Mortality in Dyspneic Patients Admitted to the Emergency Department. Dis Markers. 2020; 2020:8839506. doi: 10.1155/2020/8839506. PMID: 32655721 ; PMCID: PMC7321522.

11. Margraf A, Zarbock A. Platelets in Inflammation and Resolution. J Immunol. 2019; 2 03(9):2357-2367. doi: 10.4049/jimmunol.1900899. PMID: 31636134.

12. Siddiqui MM, Banayan JM, Hofer JE. Pre-eclampsia through the eyes of the obstetrician and anesthesiologist. Int J Obstet Anesth. 2019; 40:140-148. doi: 10.1016/j. ijoa.2019.04.002. Epub 2019 Apr 13. PMID: 31208869.

13. Laresgoiti-Servitje E, Gomez-Lopez N. The pathophysiology of preeclampsia involves altered levels of angiogenic factors promoted by hypoxia and autoantibody-mediated mechanisms. Biol Reprod. 2012; 87(2):36. doi: 10.1095/biolreprod.112.099861. PMID: 22649072. 


\section{UTILIDAD DIAGNÓSTICA DE LA RELACIÓN PLAQUETAS/LINFOCITOS EN EMBARAZADAS CON PREECLAMPSIA}

14. Gogoi P, Sinha P, Gupta B, Firmal P, Rajaram S. Neutrophil-to-lymphocyte ratio and platelet indices in pre-eclampsia. Int J Gynaecol Obstet. 2019; 144(1):1620. doi: 10.1002/ijgo.12701. Epub 2018 Nov 11. PMID: 30362112 .

15. Sitotaw C, Asrie F, Melku M. Evaluation of platelet and white cell parameters among pregnant women with Preeclampsia in Gondar, Northwest Ethiopia: A comparative cross-sectional study. Pregnancy Hypertens. 2018; 13:242-247. doi: 10.1016/j. preghy.2018.06.006. Epub 2018 Jun 9. PMID: 30177059.

16. Li H, Zhou Y, Ma Y, Han S, Zhou L. The prognostic value of the platelet-to-lymphocyte ratio in acute coronary syndrome: a systematic review and metaanalysis. Kardiol Pol. 2017; 75(7):666-673. doi: 10.5603/KP.a2017.0068. Epub 2017 Apr 10. PMID: 28394006.

17. Yavuzcan A, Cağlar M, Ustün Y, Dilbaz S, Ozdemir I, Yildiz E, et al. Mean platelet volume, neutrophillymphocyte ratio and platelet-lymphocyte ratio in severe preeclampsia. Ginekol Pol [Internet]. 2014 [consultado junio de 2020]; 85(3):197-203. PMID: 24783431. Disponible en: https://pubmed.ncbi.nlm. nih.gov/24783431/

18. Yücel B, Ustun B. Neutrophil to lymphocyte ratio, platelet to lymphocyte ratio, mean platelet volume, red cell distribution width and plateletcrit in preeclampsia. Pregnancy Hypertens. 2017; 7:29-32. doi: 10.1016/j. preghy.2016.12.002. Epub 2016 Dec 23. PMID: 28279444.

19. Kim MA, Han GH, Kwon JY, Kim YH. Clinical significance of platelet-to-lymphocyte ratio in women with preeclampsia. Am J Reprod Immunol. 2018; 80(1):e12973. doi: 10.1111/aji.12973. Epub 2018 May 21. PMID: 29781540.

20. Järemo P, Lindahl TL, Lennmarken C, Forsgren H. The use of platelet density and volume measurements to estimate the severity of pre-eclampsia. Eur J Clin Invest. 2000; 30(12):1113-8. doi: 10.1046/j.13652362.2000.00753.x. PMID: 11122327.

21. Jakobsen C, Larsen JB, Fuglsang J, Hvas AM. Platelet function in preeclampsia - a systematic review and meta-analysis. Platelets. 2019; 30(5):549-562. doi: 10.1080/09537104.2019.1595561. Epub 2019 Apr 14. PMID: 30983478.
22. Kanat-Pektas M, Yesildager U, Tuncer N, Arioz DT, Nadirgil-Koken G, Yilmazer M. Could mean platelet volume in late first trimester of pregnancy predict intrauterine growth restriction and pre-eclampsia? J Obstet Gynaecol Res. 2014; 40(7):1840-5. doi: 10.1111/jog.12433. PMID: 25056460.

23. AlSheeha MA, Alaboudi RS, Alghasham MA, Iqbal J, Adam I. Platelet count and platelet indices in women with preeclampsia. Vasc Health Risk Manag. 2016; 12:477-480. doi: 10.2147/VHRM.S120944. PMID: 27920548 ; PMCID: PMC5123587.

24. Freitas LG, Alpoim PN, Komatsuzaki F, Carvalho Md, Dusse LM. Preeclampsia: are platelet count and indices useful for its prognostic? Hematology. 2013; 18(6):360-4. doi: 10.1179/1607845413Y.0000000098. Epub 2013 May 10. PMID: 23676885.

25. Wang J, Zhu QW, Cheng XY, Liu JY, Zhang LL, Tao YM, et al. Assessment efficacy of neutrophillymphocyte ratio and monocyte-lymphocyte ratio in preeclampsia. J Reprod Immunol. 2019; 132:29-34. doi: 10.1016/j.jri.2019.02.001. Epub 2019 Mar 6. PMID: 30861482.

26. Fogerty AE. Thrombocytopenia in Pregnancy: Mechanisms and Management. Transfus Med Rev. 2018; 32(4):225-229. doi: 10.1016/j.tmrv.2018.08.004. Epub 2018 Aug 23. PMID: 30177431.

27. Yılmaz ZV, Yılmaz E, Küçüközkan T. Red blood cell distribution width: A simple parameter in preeclampsia. Pregnancy Hypertens. 2016; 6(4):285-287. doi: 10.1016/j.preghy.2016.05.001. Epub 2016 May 6. PMID: 27939469.

28. Kalafat E, Thilaganathan B. Cardiovascular origins of preeclampsia. Curr Opin Obstet Gynecol. 2017; 29(6):383-389. doi: 10.1097/GCO.0000000000000419. PMID: 28961633.

29. Tomimatsu T, Mimura K, Matsuzaki S, Endo M, Kumasawa K, Kimura T. Preeclampsia: Maternal Systemic Vascular Disorder Caused by Generalized Endothelial Dysfunction Due to Placental Antiangiogenic Factors. Int J Mol Sci. 2019; 20(17):4246. doi: 10.3390/ijms20174246. PMID: 31480243; PMCID: PMC6747625

Recibido 13 de agosto 2020 Aprobado 10 de noviembre de 2020 